\title{
Vascular corrosion casting: analyzing wall shear stress in the portal vein and vascular abnormalities in portal hypertensive and cirrhotic rodents
}

\author{
Christophe Van Steenkiste ${ }^{1}$, Bram Trachet ${ }^{2}$, Christophe Casteleyn ${ }^{3}$, Denis van Loo ${ }^{4}$, Luc Van Hoorebeke ${ }^{4}$, \\ Patrick Segers ${ }^{2}$, Anja Geerts ${ }^{1}$, Hans Van Vlierberghe ${ }^{1}$ and Isabelle Colle ${ }^{1}$
}

\begin{abstract}
Vascular corrosion casting is an established method of anatomical preparation that has recently been revived and has proven to be an excellent tool for detailed three-dimensional (3D) morphological examination of normal and pathological microcirculation. In addition, the geometry provided by vascular casts can be further used to calculate wall shear stress (WSS) in a vascular bed using computational techniques. In the first part of this study, the microvascular morphological changes associated with portal hypertension (PHT) and cirrhosis in vascular casts are described. The second part of this study consists of a quantitative analysis of the WSS in the portal vein in casts of different animal models of PHT and cirrhosis using computational fluid dynamics (CFD). Microvascular changes in the splanchnic, hepatic and pulmonary territory of portal hypertensive and cirrhotic mice are described in detail with stereomicroscopic examination and scanning electron microscopy. To our knowledge, our results are the first to report the vascular changes in the common bile duct ligation cirrhotic model. Calculating WSS using CFD methods is a feasible technique in PHT and cirrhosis, enabling the differentiation between different animal models. First, a dimensional analysis was performed, followed by a CFD calculation describing the spatial and temporal WSS distributions in the portal vein. WSS was significantly different between sham/cirrhotic/pure PHT animals with the highest values in the latter. Up till now, no techniques have been developed to quantify WSS in the portal vein in laboratory animals. This study showed for the first time that vascular casting has an important role not only in the morphological evaluation of animal models of PHT and cirrhosis, but also in defining the biological response of the portal vein wall to hemodynamic changes. CFD in 3D geometries can be used to describe the spatial and temporal variations in WSS in the portal vein and to better understand the forces affecting mechanotransduction in the endothelium.
\end{abstract}

Laboratory Investigation (2010) 90, 1558-1572; doi:10.1038/labinvest.2010.138; published online 16 August 2010

KEYWORDS: cirrhosis; CFD; PHT; vascular corrosion casting; WSS

Portal hypertension (PHT), liver cirrhosis and their associated complications are the major causes of morbidity and mortality in the Western world. ${ }^{1}$ Our knowledge of the pathogenesis and molecular mechanisms of PHT has dramatically increased in recent years. ${ }^{2}$ In particular, the process of angiogenesis, defined as the growth of new blood vessels from a preexisting vascular tree, and its role in modulating hepatic resistance, portosystemic collateral formation and increased splanchnic blood flow have become the hallmark pathological features of PHT and cirrhosis. ${ }^{3}$ A variety of methods has been described to obtain valid representations of this newly formed vasculature via in vivo medical imaging (mostly MRI and CT) $)^{4,5}$ and on post-mortem preparations (including in situ vascular corrosion casting). ${ }^{6}$

To increase our understanding of these pathologies, the morphological changes should be described with as much detail as possible, but one might also consider the hemodynamic conditions that may be a driving force in the formation of new blood vessels. Vascular corrosion casting has recently revived and has proven to be an excellent tool for detailed three-dimensional (3D) morphological examination of normal and pathological microcirculation. ${ }^{5,7}$ In addition, the geometry provided by these vascular casts can be further used to calculate wall shear stress (WSS) in the arterial

\footnotetext{
${ }^{1}$ Department of Hepatology and Gastroenterology, Ghent University Hospital, Ghent, Belgium; ${ }^{2}$ bioMMeda, IBiTech, Ghent University, Ghent, Belgium; ${ }^{3}$ Department of Morphology, Faculty of Veterinary Medicine, Ghent University, Merelbeke, Belgium and ${ }^{4}$ Centre for X-Ray Tomography, Ghent University, Ghent, Belgium Correspondence: Dr C Van Steenkiste, MD, Department of Hepatology and Gastroenterology, Ghent University Hospital, building K12 first floor IE, De Pintelaan 185, Ghent 9000, Belgium. E-mail: Christophe.vansteenkiste@UGent.be
} 
vascular bed using computational techniques. When blood is in motion, a stress is applied parallel or tangential to the vessel wall, defined as WSS (unit: pascal). ${ }^{8}$ It has been shown that the mechanical forces applied to vascular walls induce a number of complex cell reactions and modulate gene expression and the secretion of several biomolecules. ${ }^{9}$ Furthermore, WSS calculations have recently been shown to provide prognostic data in the field of cardiovascular research. ${ }^{10,11}$

Although complicated, techniques for the in vivo determination of WSS in the arterial circulation (based on measurement of the flow velocity) exist. However, to our knowledge, these are rarely used in the venous vascular tree and have never been applied in the setting of PHT. In normal circumstances, venous WSS ranges are considerably low, ${ }^{8}$ but they increase in regions with vascular geometries that promote turbulent flow or increased flow velocity (eg, anastomoses and bifurcations) and therefore, by extension, they can also be applied in the field of PHT.

The aim of this study was to characterize the morphological vascular features and to assess the associated changes in WSS in the portal vein in different animal models of PHT and cirrhosis. The first part of this study focuses on the microvascular morphological changes associated with PHT and cirrhosis. To our knowledge, the obtained results are the first to describe the vascular changes in common bile duct cirrhosis (common bile duct ligation (CBDL)). The second part of this study consists of a quantitative analysis of the WSS in the portal vein in casts of different animal models using computational fluid dynamics (CFD) methods. Provided with the correct fluid characteristics, such as the inlet blood flow of the portal vein, and in combination with geometrical features, these numerical models can accurately predict the WSS in these animal models. ${ }^{12}$

\section{MATERIALS AND METHODS Animals}

In the first morphological part of the study, male 50\% Sv129/ $50 \%$ Swiss mice (5-8 weeks old) were purchased from Harlan Laboratories (Horst, the Netherlands). Although the vascular corrosion casts of these mice were perfectly suited to study changes in the microvasculature, it was difficult to reconstruct reliable 3D models for the second hemodynamic part of the study (ie, WSS measurements). Owing to their larger anatomical dimensions, 11 rats (Wistar, $\pm 200 \mathrm{~g}, 6-7$ weeks old, Harlan laboratories) were used for the CFD analysis in the second part of the study. The animals were kept at a constant temperature and humidity in a 12 -h controlled light/dark cycle. The institutional ethical review board at the Faculty of Medicine and Health Sciences, Ghent University, Belgium, approved the protocols.

\section{Mouse and Rat Models of PHT and Cirrhosis}

For the first morphological part of this study, mice were used to describe the anatomy, whereas the WSS measurements in the second part of this paper were performed on rats.
A model of secondary biliary cirrhosis (CBDL) and a model for pure PHT (partial portal vein ligation (PPVL)) were induced as previously described. ${ }^{13}$

Briefly, under inhalation anesthesia with isoflurane (Abbott, Louvain-la-Neuve, Belgium), the common bile duct was occluded with a double ligature of a nonresorbable suture (silk cut 7-0) in CBDL. The bile duct was then resected between the two ligatures. In PPVL, a calibrated stenosis of the portal vein was performed by placing a ligature (silk cut 5-0) around both the portal vein and an adjacent 27-gauge blunt-tipped needle. Subsequent removal of the needle yielded a calibrated constriction of the portal vein.

For the morphological study, experiments were performed 14 days after induction in the PPVL mice $(n=9)$ and 6 weeks after induction in the CBDL mice $(n=8)$. Sham-operated (SO) mice $(n=6)$ were used as controls. Previous studies have demonstrated PHT and a high degree of portosystemic collaterals 2 weeks after PPVL and 6 weeks after CBDL. ${ }^{14,15}$

For the WSS analysis, vascular corrosion casts were obtained in four PPVL, four CBDL and three SO rats, and experiments were performed on the same time points as described above.

Mortality rates after PPVL, CBDL and sham induction were 5,10 and $0 \%$, respectively, and were similar to earlier studies. ${ }^{13}$

\section{Assessing WSS from CFD in the Portal Vein of Rats: Workflow}

The workflow followed to obtain WSS values can be summarized as follows:

(1) defining the 3D geometry by casting (Vascular Corrosion Casting),

(2) dividing the volume occupied by the fluid in a mesh (Building a 3D Model of a Rat Cast Specimen),

(3) defining the physical modeling (using the Navier-Stokes equations for the flow (Numerical Simulations of Blood Flow, Using CFD Analysis in the Portal Vein of Rats)),

(4) defining the boundary conditions (by specifying the flow velocities at the boundaries of the geometry (Hemodynamic Measurements)) and

(5) solving the equations (Numerical Simulations of Blood Flow, Using CFD Analysis in the Portal Vein of Rats).

These items are further clarified in the indicated paragraphs.

\section{Vascular Corrosion Casting}

Following 24-h fasting, animals were killed by intraperitoneal (i.p.) injection of phenobarbital $(150 \mathrm{mg} / \mathrm{kg}$ ) (Nembutal, Ceva Sante Animale, Brussels, Belgium) after proper anesthesia (ketamine $(100 \mathrm{mg} / \mathrm{kg}$ body weight i.p.; Ketalar, Pfizer, Brussels, Belgium) and xylazine $(10 \mathrm{mg} / \mathrm{kg}$ body weight; i.p., Rompun, Bayer, Brussels, Belgium)). A midline abdominal incision was performed, and a 26-gauge catheter (Terumo, Leuven, Belgium) was inserted into the ileocolic vein to cast the liver/splanchnic microvasculature $(n=6$ for 
PPVL mice, $n=4$ for CBDL mice and $n=3$ for sham mice, all rats) and into the inferior caval vein to cast the lung microcirculation ( $n=3$ for PPVL mice, $n=4$ for CBDL mice and $n=3$ for sham mice).

For each cast, fresh Batson's solution was prepared using Batson's \#17 corrosion kit (Brunschwig chemie, Amsterdam, the Netherlands) according to the manufacturer's recommendations. ${ }^{16}$ Either 2 or $20 \mathrm{ml}$ of Batson's solution were injected in mice and rats, respectively, through the catheter using 1-ml tuberculin syringes. Animal bodies were immersed for $30 \mathrm{~min}$ in tepid water during polymerization and macerated overnight in $25 \%$ potassium hydroxide. The vascular corrosion casts were rinsed gently for $3 \mathrm{~h}$ with streaming water. Blood vessels were evaluated and photographed with a stereomicroscope (Olympus SZX7, Olympus Belgium, Aartselaar, Belgium) equipped with a chargecoupled device camera (Olympus Colorview, Olympus Belgium). Subsequently, samples of the casts were mounted on a metal stub, platinum-coated (Jeol auto fine coater, JFC 1300, Zaventem, Belgium) and examined by a scanning electron microscope (Jeol JSM $5600 \mathrm{LV}$ ). For the first morphological part of the study, the liver/lungs/mesentery of the mice were systematically examined in the different models, but only the particularities were recorded and illustrated in figures.

\section{Morphometric Analysis on Vascular Corrosion Casts of Mice}

In order to provide a systematic description of the electron microscopic images, quantitative information was derived from the vascular corrosion casts. The electron microscopic images were characterized morphometrically, taking into account the different issues that can limit a quantitative analysis on casts (Supplementary Methods S1). The samples were screened by scanning electron microscopy at a low magnification $(\times 70)$ to detect the two most vascularized areas (hot spots). Pictures were taken of these hot spots at the appropriate magnification $(\times 500$ to $\times 1000)$ in those areas positioned in a single horizontal plane perpendicular to the orientation of the electron beam (to eliminate false readouts at slightly inclined vessels). The capillary diameter, as a marker of the microvascular dilation in the lung, and the intercapillary distance, as an indicator of the vascular density in the mesentery, were measured quantitatively using computerized image analysis (Cell D, Olympus Soft Imaging Solutions, Münster, Germany). In order to measure a straight line segment as the true diameter (and to avoid skewed oblique distances), measurements of the capillary diameters were performed perpendicular to the longitudinal axis of the vessel wall (Supplementary Methods S2.1.). All intercapillary distances were determined in the mesenteric tributaries with approximately the same vessel size. Standardized measurements were taken between the different ramifications at the base of a long straight capillary mesenteric segment (Supplementary Methods S2.2.). The mean values of all measurements per picture (two pictures per mouse) were determined and averaged with the mean capillary diameter/ intercapillary distance of three mice per group. Results were expressed as the mean $\mu \mathrm{m} \pm$ s.e.m.

\section{Immunohistochemistry: CD31 Staining}

Sections from paraffin-embedded tissues (mesentery (three loops proximal to the cecum), liver and lung tissues) of mice were incubated with anti-CD31 antibodies (Supplementary Methods S3). Antigen localization was visualized with $3^{\prime}$-3-diamino benzidene as chromogen. Counterstaining was performed with hematoxylin. These CD31 stainings were correlated with the changes observed on the vascular corrosion casts.

\section{Hemodynamic Measurements}

Hemodynamic parameters were recorded before the injection of Batson's solution. Blood pressure was measured using a pneumatic pulse transducer (Marco Bio-systems, Houston, TX, USA) in conjunction with a PowerLab system (AD Instruments, Oxfordshire, UK). To obtain flow data for WSS analysis in rats, an ultrasonic blood flow probe (Transonic Systems, Ithaca, NY, USA) was placed around the portal vein, just above the level of the splenic and mesenteric vein confluence, thereby allowing measurement of the portal venous inflow $(\mathrm{ml} / \mathrm{min})$.

\section{Dimensional Analysis in the Portal Vein of Rats}

To reveal a trend and rapid snapshot of the magnitude of WSS, a dimensional analysis was performed, yielding results that were averaged over time and space. However, more accurate and precise results were obtained by CFD analysis afterward by taking the $3 \mathrm{D}$ geometry and the temporal and spatial variations of the shear stress patterns into account (vide infra). ${ }^{12,17}$

A critical input to the dimensional analysis was the mean Reynolds number (Re), which is defined in more detail in Appendix A. The Re is a key dimensionless parameter in fluid dynamics, which expresses the ratio of inertial and viscous forces. It allows the assessment of whether flow is laminar $(\operatorname{Re}<2000)$, turbulent $(\operatorname{Re}>2500)$ or in a transition phase $(2000 \leq \operatorname{Re} \leq 2500)$. Assuming that the kinematic blood viscosity $(v)$ is constant, the Reynolds number is calculated using the mean inlet velocity $(V)$ and mean inlet portal vein diameter of each model. The WSS is computed using Poiseuille's law $^{18}$ (Appendix A). However, this formula is only valid in laminar steady flow conditions in a straight cylindrical tube and not in more physiological situations. Taking the pulsatile blood flow into account, another dimensionless expression of pulsatile flow frequency in relation to viscous effects is introduced, defined as the Womersley number $(\alpha)$. When $\alpha$ is low, the WSS can be estimated by Poiseuille's law (Appendix A). 


\section{Building a 3D Model of a Rat Cast Specimen}

The 3D geometry of the cast was acquired with an in-house developed $\mu$-CT. The setup consists of a closed-type X-ray tube with directional target (Hamamatsu Photonics KK, Shimokanzo, Japan) and an A-Si flat panel detector (Varian Medical Systems, Palo Alto, CA, USA). The detector was used in binning 2 mode $(0.254 \mu \mathrm{m}$ pixel size) and 1200 projection images were recorded at $1 \mathrm{~s}$ per image while the cast was rotated over $360^{\circ}$. The tube was operated at $80 \mathrm{kV}$ and $180 \mu \mathrm{A}$ with a magnification of $3.2(\mathrm{FOV}=6 \mathrm{~cm})$, as previously described. ${ }^{12}$ The reconstruction was performed with Octopus software (Ghent University, Ghent, Belgium), resulting in a $700 \times 700 \times 700$ voxels data set with $79 \mu \mathrm{m}$ voxel pitch. The obtained images were segmented with Mimics software (Materialise, Leuven, Belgium) to produce a 3D computer model of the portal vein starting just downstream of the level of the splenic vein and including the left and right portal branches. The scanned geometry contained multiple small irregularities, which were trimmed and surface smoothed to create a computational model. The original model was then automatically rescaled according to a simultaneously scanned digital meter scale. To perform blood flow simulations, each model had to be meshed or subdivided into a finite number of small volume elements. Such a volume mesh was created in the TGrid software (Ansys, Canonsburg, PA, USA), and consisted of a boundary layer with prismatic elements near the wall to improve accuracy where it was most needed. The bulk of the model was filled with tetrahedral elements. For 3D computer modeling, an infinite number of volume elements should theoretically be used to exactly approximate the underlying geometry. However, in a numerical study, only a finite number is applicable with the necessity to trade off between physical adequacy and numerical efficiency. In order to address this problem, a mesh-independent study was performed on the original model. We changed the number of volume elements from $5 \times 10^{5}$ to $18 \times 10^{5}$ and the analysis showed that the resulting $95 \%$ value (WSS95\% as a marker of highest WSS values; only $5 \%$ of WSS values are higher than this value) and the spatially averaged WSS (WSSav) remained constant for models containing $12 \times 10^{5}$ elements or more. A mesh density of $12 \times 10^{5}$ elements was therefore used in all models.

\section{Numerical Simulations of Blood Flow, Using CFD Analysis in the Portal Vein of Rats}

After having built a 3D volume mesh, CFD software (Fluent, Ansys) was used to numerically solve the Navier-Stokes equations for the flow across the 3D geometry. To calculate a realistic flow field, boundary conditions are needed to provide information on the flow behavior at the in- and outlets of the model. These boundary conditions should ideally be obtained via in vivo measurements of pressure and/or flow at these locations. We imposed a time-dependent velocity profile at the inlet of our model (the inlet of the portal vein) that was calculated from a flow profile measured just downstream of the level of the splenic vein (Hemodynamic Measurements). The flow split at all side branches was calculated using Murray's law, stating that the ratio between the flows to each branch is proportional to the third power of the ratio of the branch diameters. This resulted in an average flow split of $35 \pm 8 \%$ into the left and $65 \pm 8 \%$ into the right portal vein, which agrees well with the values obtained by Teleuhan et al, ${ }^{19}$ who derived a flow split of 70 and $30 \%$ into the right and left portal veins, respectively. Rat blood density was taken to be $1060 \mathrm{~kg} / \mathrm{m}^{3}$ and the dynamic blood viscosity was assumed to be a constant asymptomatic value of $3.5 \mathrm{mPa} .{ }^{17,20}$ The measured inlet profile was divided into 40 time steps, and three cardiac cycles for each of the 40 time steps were calculated. The resulting data of the last cycle were then post-processed in Tecplot (Bellevue, WA, USA). Owing to major differences in the portal vein diameters of two rats (one sham and one PPVL, in comparison with other subjects in the respective groups) resulting from differences in the body weights of these rats at the induction of the model, we decided to rescale these two diameters into the mean portal vein inlet diameters of the sham and PPVL groups, respectively, as was described earlier in previous studies. ${ }^{12}$

To quantify the difference in WSS between the models, WSSav and WSS95\% were computed for each time-averaged model (Appendix A), as well as the oscillatory shear index (OSI) distribution. The latter was introduced to analyze the degree of oscillation: OSI is 0 if WSS always acts in the same direction. However, if WSS is oscillating between positive and negative values, OSI will increase, with 0.5 as a theoretical maximum. $^{21,22}$ As endothelial cells are susceptible to the magnitude and changes in WSS direction, high WSSav and OSI values are indirect markers for disturbed endothelial function. ${ }^{12,23}$ The spatial distribution of the WSS and OSI (across the wall of the portal vein, time averaged over the total cardiac cycle) and also the temporal variation in WSS (at different time points throughout the cardiac cycle) were calculated and their distributions were plotted.

\section{Statistics}

Data analysis was performed using SPSS version 15.0 (SPSS, Chicago, IL, USA). In cases of normally distributed data, groups were compared with the Student's $t$-test for independent samples. For other types of data, the MannWhitney $U$-test was performed. Data are presented as the mean \pm s.e.m. or median \pm range. $P$-values $<0.05$ (two-tailed probability) were regarded as significant.

\section{RESULTS \\ Vascular Architecture in PHT and Cirrhosis in Mice Splanchnic architecture}

Splenorenal shunts were visualized in PPVL mice (Figure 1a). As shown in Figure 1a, a large collateral vessel branched off from the splenic vein and anatomized with the left renal vein. In addition, a newly formed vascular network with irregular, tortuous and immature vessels could be detected as an 

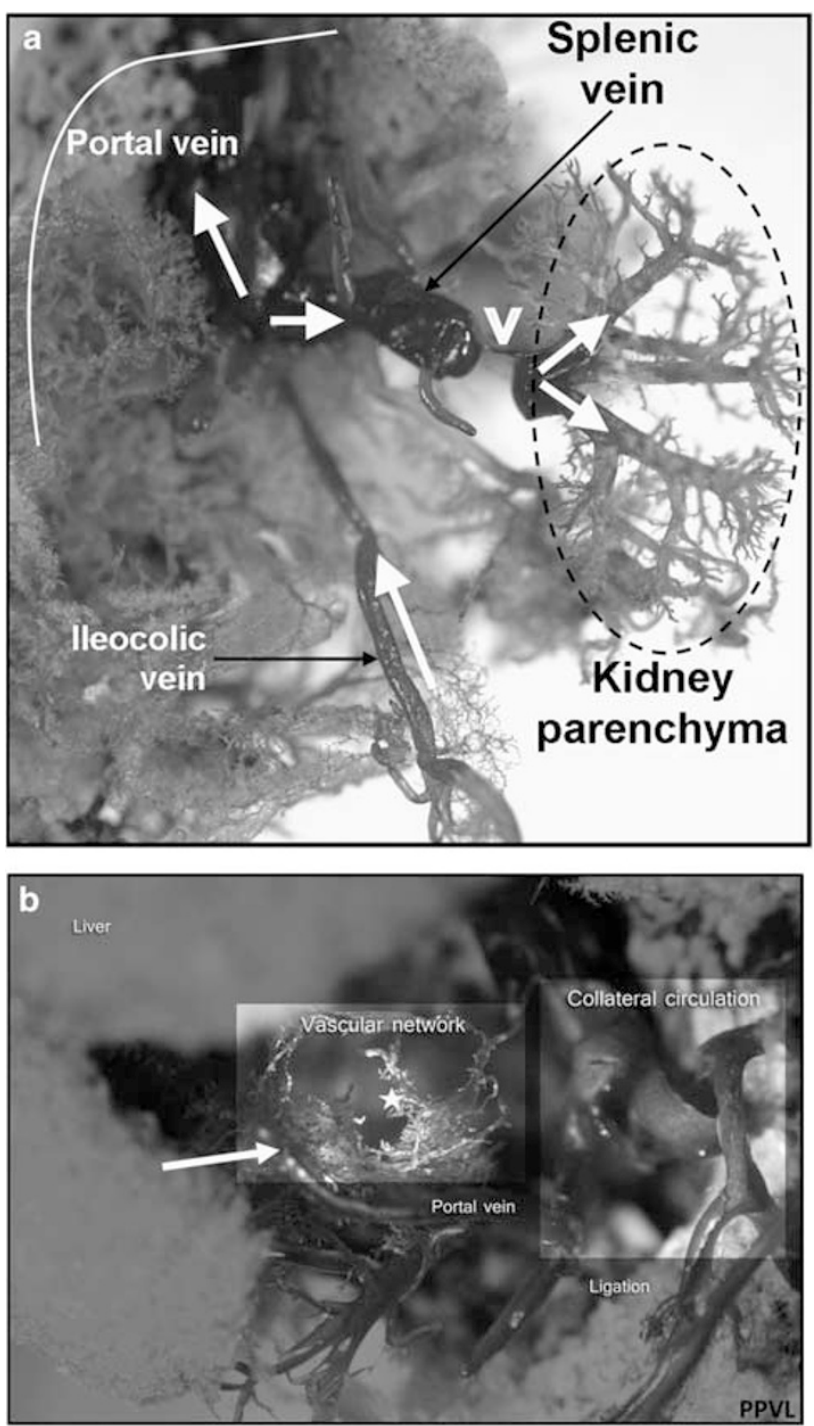

Figure 1 Splanchnic vascular cast images of PPVL mice after the injection of Batson's solution into the ileocolic vein. (a) Presence of extrahepatic splenorenal shunt (white arrow head) between the splenic vein and the left renal vein. Note the retrograde filling of the shunt with subsequent demonstration of the dichotomic venous branching of the kidney. The spleen and parts of the liver (dotted line) have been removed to optimize the visibility of the shunt. The flow direction of the Batson's solution has been indicated by the large white arrows. (b) View on a newly formed vascular network with irregular, tortuous and immature vessels (star) between branches of the portal vein (white arrow) and the inferior caval vein (not shown on this image). As indicated, portal vein ligation was associated with numerous tortuous collaterals, bypassing this ligation and favoring the direction of Batson's solution into the route of minimum resistance, that is, the collateral circulation. Small branches of the newly formed vascular network and collaterals are also extending to the inferior caval vein, but because of the dorsal position of this vein these branches are not visible on this figure.

extrahepatic shunt between the portal vein branches and the inferior caval vein (Figure $1 \mathrm{~b}$ ).

In all PPVL and CBDL mice, the splanchnic vascular density was significantly higher than that in SO mice (Figures 2a, b and e (sham) vs Figures $2 \mathrm{c}, \mathrm{d}$ and $\mathrm{f}$ (PPVL)).
This was also reflected by the morphometric analysis of the intercapillary distance, which is inversely correlated with the vascular density. The intercapillary distance was significant larger in the SO animals $(145.3 \pm 10.9 \mu \mathrm{m})$ compared with the PPVL $(57.4 \pm 25.9 \mu \mathrm{m}, P<0.05 v s$ sham $)$ and CBDL animals (18.9 $\pm 7.0 \mu \mathrm{m}, P<0.01$ vs sham), reflecting the higher vascular density in portal hypertensive and cirrhotic mice.

The splanchnic microvasculature network in PPVL/CBDL mice consisted of multiple vessels with irregular and chaotic branching, numerous blind ends and a lack of hierarchy (Figures $2 \mathrm{c}$ and $\mathrm{d}$ ). This architecture clearly differed in many features from the vasculature of normal tissues (Figures 2a and b). In addition, sprouting angiogenesis was observed more frequently in PPVL animals (Figures $2 \mathrm{~g}$ and Supplementary Results S1A).

Remarkably, the vessel branches displayed small holes on their walls in PPVL, serving as a marker for intussusceptive capillary growth and representing one of the two principles of microvascular growth, namely nonsprouting angiogenesis (Figure 2h). Venules were identified by their characteristic endothelial cell nuclear imprints (superficial, poorly contoured and irregularly dispersed through the surface) (Supplementary Results S1B). The vasa vasorum externae, which supply the larger veins, were observed as cord-like structures, stretching over and finally penetrating the vessel wall (Supplementary Results S1A). The number of the vasa vasorum in the larger splanchnic veins was not different in PPVL/CBDL animals compared with sham animals. As splanchnic neoangiogenesis in PPVL/CBDL is predominantly present in medium-sized vessels and capillaries, the net effect on the amount of vasa vasorum (in larger vessels) after ligation might be less prominent.

\section{Hepatic architecture}

Scanning electron microscopy of CBDL livers revealed marked microvascular changes in comparison with sham livers. Casts of control livers showed normal filling of the sinusoids with a trabecular pattern between columns of hepatocytes (Figure 3a). At the fibrous capsule of Glisson, the sinusoids were not entirely filled with Batson solution, resulting in a Christmas tree appearance with an abundance of small-sized branches encircling the portal venules (Figure $3 \mathrm{~b}$ ). In contrast, CBDL livers displayed a complete loss of architecture with localized obliteration of sinusoids (Figures $3 \mathrm{c}$ and d). Note the occurrence of holes in the cast due to bilomas (Figure 3c). By consequence, the irregular, disrupted, bulging and saccular 3D organization of the sinusoids did not allow a more exhaustive morphometric analysis on the hepatic vascular bed.

Large portocentral collaterals that bypassed the capillaries and shunted blood directly to the hepatic veins often appeared in CBDL livers. Small globular extravasations, sticking locally to these collateral vessels, occurred as leakage of the casting media (Figures $3 \mathrm{c}$ and $\mathrm{d}$ ). 

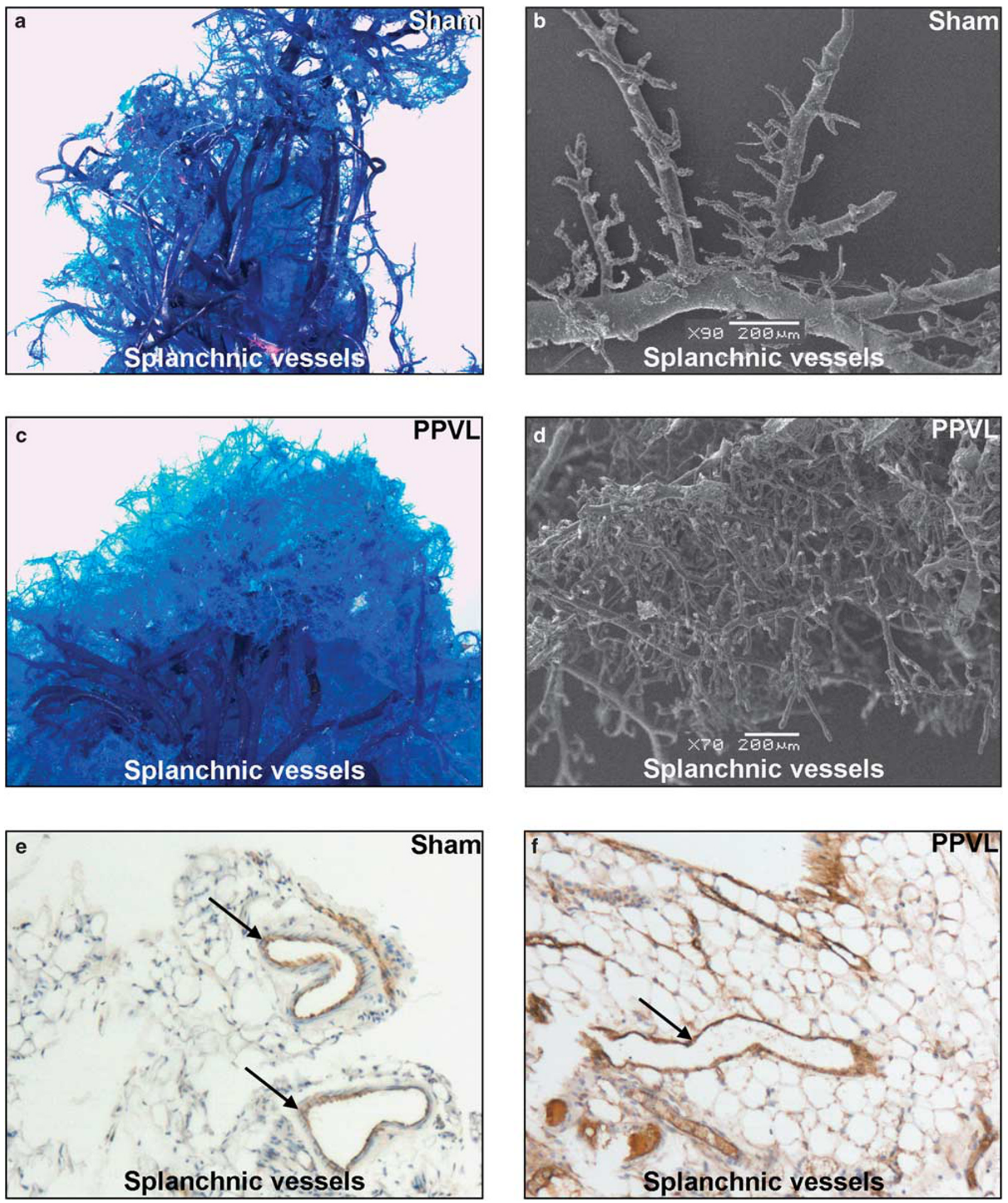

Figure 2 Splanchnic vascular cast images of PPVL and sham mice after the injection of Batson's solution into the ileocolic vein. Representative stereomicroscopic and scanning electron microscopic photographs of the mesentery showing increased splanchnic neoangiogenesis in PPVL mice (c, d) in comparison with sham mice $(\mathbf{a}, \mathbf{b})$, as also confirmed on CD31 immunohistochemistry (e, $\mathbf{f}$, arrows indicate blood vessels). Sprouting angiogenesis (g, arrows) was observed more frequently in PPVL than in sham mice. Illustration of intussusceptive angiogenesis in PPVL mice is additionally shown (h, arrow). 


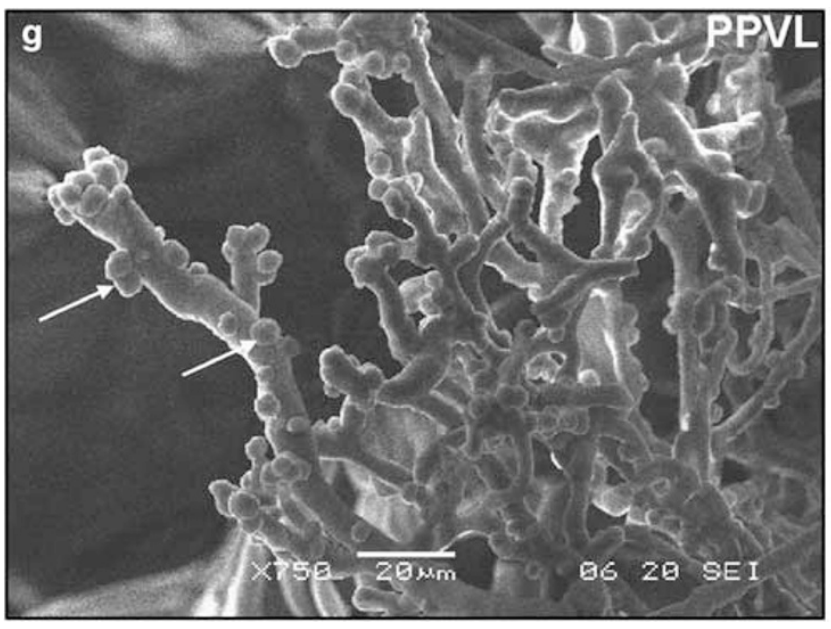

Figure 2 Continued.

In comparison, biliary hyperplasia and vascular proliferation were also present on CD31 immunohistochemistry in CBDL (Figures $3 e$ and $\mathrm{f}$ ).

\section{Pulmonary architecture}

In the normal pulmonary circulation, the capillary arborization is organized into a honeycomb-like network (Figure 4a). The morphology has always been described in both inferior lung lobes, as vascular density is more pronounced therein. Scattered lumpy regions could be recognized due to excessive pressure and rupture of the alveoli in normal and cirrhotic casts (eg, Figure 4b). Cirrhotic mice displayed the same vascular pattern, but larger vessels often appeared in the inferior lobes of cirrhotic mice, shunting blood directly to the lung veins without passing the alveoli (Figures $4 \mathrm{~b}$ and $\mathrm{f}$ ). Interestingly, intussusceptive capillary growth was observed more frequently in CBDL mice, although it was also present in normal lungs, as previously described in the development of the normal pulmonary microcirculation in rats (Figures $4 \mathrm{c}$ and $\mathrm{d}$ ). ${ }^{24}$ The different steps in the generation of new vascular segments by intussusception were clearly illustrated: protrusion of a capillary pillar from opposite sides of the vessel wall into the vascular lumen is followed by perforation of the vessel (Figure 4c) and, ultimately, the splitting into two new vessels (Figure 4d). ${ }^{25}$

Previous work ${ }^{26}$ already indicated that the CBDL model is the only established experimental model that reproduces the physiological features of human hepatopulmonary syndrome (HPS), whereas other commonly used models, such as PPVL, do not result in the development of HPS. As the hallmark feature of the HPS is a microvascular dilatation occurring in the pulmonary artery circulation, the lung capillaries in CBDL/PPVL and sham mice were hereby characterized morphometrically. The capillary width ranged from $7.82 \pm 0.29 \mu \mathrm{m}$ in CBDL animals to $6.95 \pm 0.25 \mu \mathrm{m}$ in PPVL animals, compared with $6.20 \pm 0.51 \mu \mathrm{m}$ in sham mice, confirming the significant microvascular dilatation after CBDL

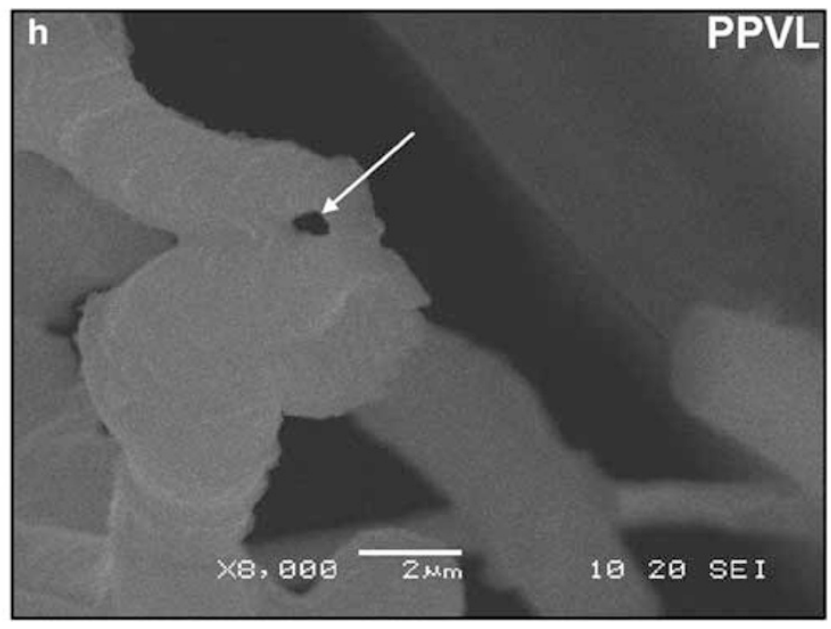

$(P<0.05 v s$ sham $)$. These findings confirm earlier work from Fallon $e t a l^{26}$ and Schraufnagel et $a l^{27}$ showing pulmonary vasodilation in CBDL, but not in PPVL and sham animals.

\section{Analysis of WSS in Different Rat Models of PHT and Cirrhosis \\ Dimensional analysis}

Representative measured flow waveforms $(\mathrm{ml} / \mathrm{min}$ ) for the portal venous inflow are shown in Figure 5. The flow rates in the PPVL and CBDL rats were significantly higher in comparison with those in the $\mathrm{SO}$ rats $(1.54,1.59,0.91 \mathrm{ml} / \mathrm{min}$, respectively; $P<0.01$ PPVL $v s$ sham, $P<0.05$ CBDL $v s$ sham). The results from the dimensional analysis are summarized in Table 1 . The Re at the inlet of the model, indicating whether flow is laminar/turbulent or in transition phase, increased from $115 \pm 17$ in sham rats to $285 \pm 27$ and $237 \pm 6$ in CBDL and PPVL rats, respectively $(P<0.01 v s$ sham and $P<0.001$ $v s$ sham). The Womersley numbers, taking the pulsatile blood flow into account, varied from 2.9 in the SO rats to 4.1 in the PPVL rats. Although only a trend in WSS can be detected by the dimensional analysis (as idealized Poiseuille flow is assumed), the markedly higher WSS values in PPVL/CBDL rats $v s$ sham were already obvious $(P<0.05$, both $v s$ sham, Table 1). Importantly, no differences in WSS were shown between PPVL and CBDL.

\section{CFD}

CFD analysis allowed us to describe the spatial and temporal variations of WSS in more detail. The spatial distribution of WSS (time averaged over the total cardiac cycle) along the course of the portal vein is shown in different animal models in Figure 6. From an anatomical perspective, the portal vein divides into two large branches, the left and right portal veins, and ramifies further, forming smaller venous vessels. Another tributary of the portal vein is the left gastric vein (or coronary vein), originating just proximal from the left/right portal confluence. In physiological situations, the coronary vein may be occluded or exhibits only a very marginal blood 

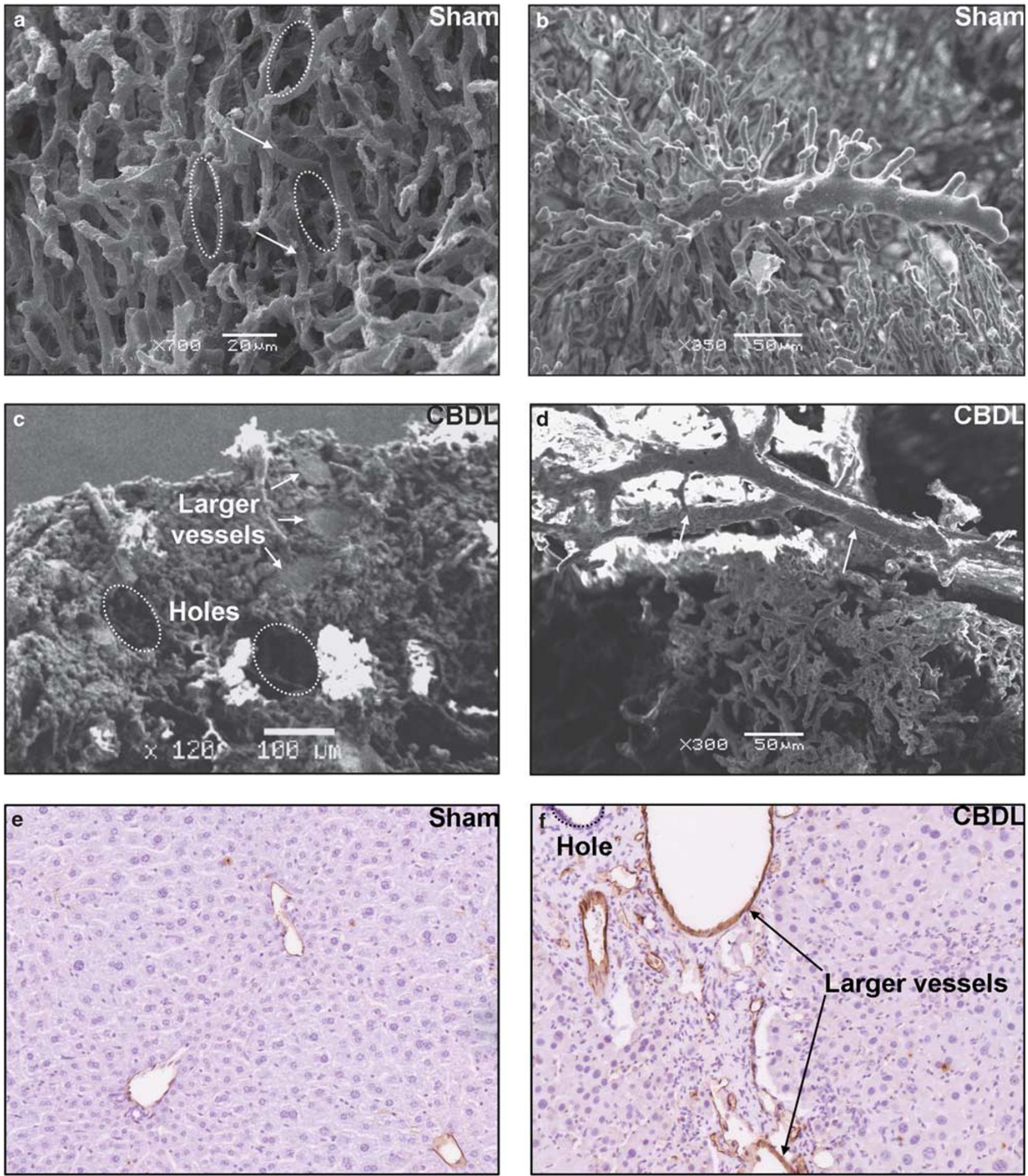

Figure 3 Liver vascular cast images of CBDL and sham mice after the injection of Batson's solution into the ileocolic vein. Casts of control livers showing normal reticular filling of the sinusoids (a, arrows) with liver cell columns (a, circles) between the sinusoids. At the liver capsule, the sinusoids were not entirely filled with Batson's solution, resulting in a Christmas tree appearance with an abundance of small-sized branches encircling the portal venule (b). In contrast, CBDL livers displayed a complete loss of architecture with localized obliteration of sinusoids, leaving a remnant and damaged microcirculation (c). Note the occurrence of holes in the cast due to bilomas (c, circles). Portocentral collaterals are present in the liver, bypassing the sinusoids and shunting blood directly to the hepatic veins (c, $\mathbf{d}$, arrows). These shunts exhibit a leaky appearance, reflecting changes in vascular permeability and yielding easily to leakage of the casting media (d). Representative light microscopic images of CBDL livers (f) confirm the expansion of the vascular structures (larger vessels) and the biliary hyperplasia (holes) compared with sham mice (e). 

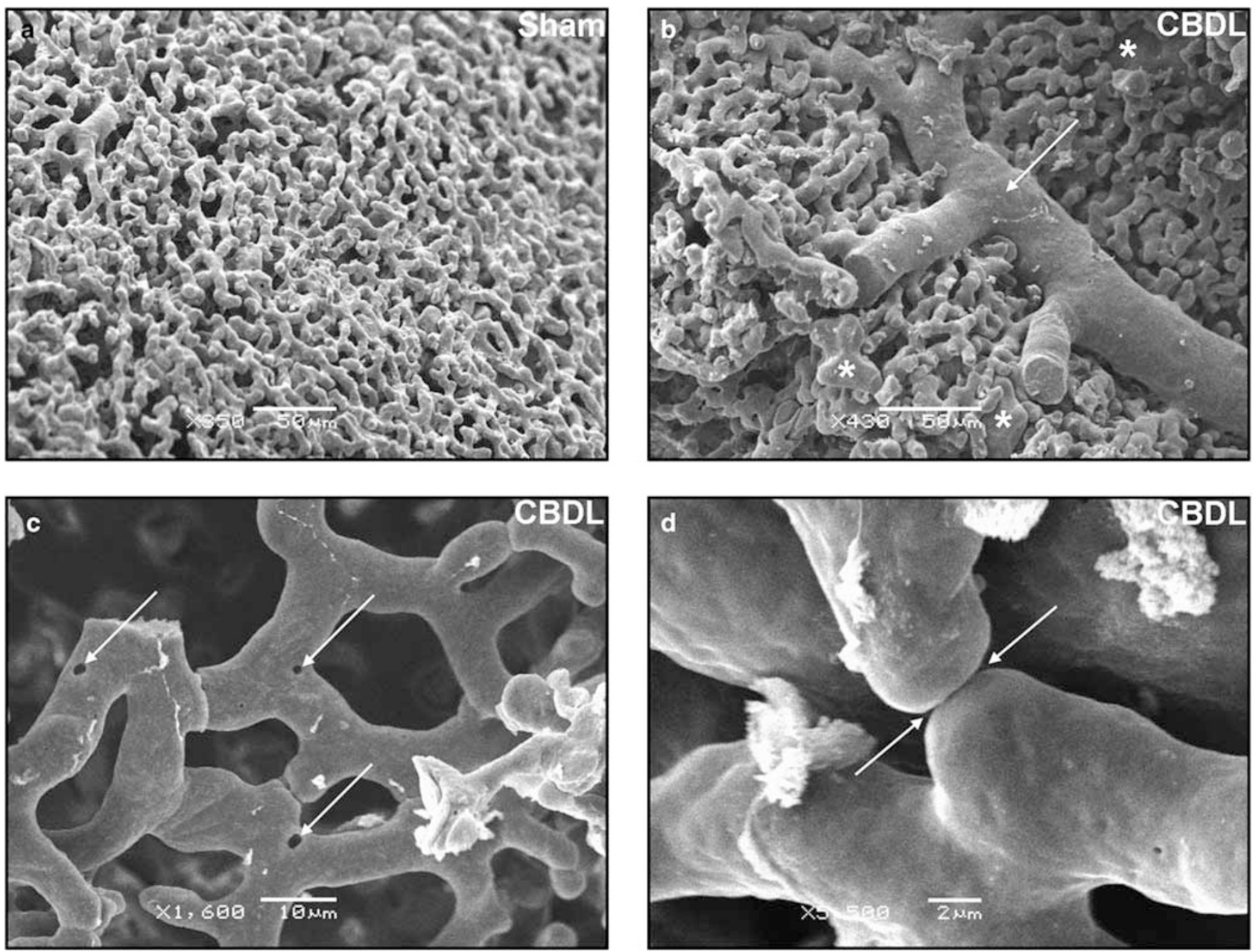

Figure 4 Pulmonary vascular casting images of CBDL mice after the injection of Batson's solution into the inferior caval vein. Casts from normal pulmonary vasculature at the pleural surface showed a vascular arborization, forming honeycomb-like vascular networks (a). Large vessels often appeared in the inferior lobes of cirrhotic mice, shunting blood directly to the lung veins without passing the alveoli (arrow) (b). Scattered lumpy regions, which are due to excessive pressure and rupture of the alveoli (white asterisks) could be recognized (b). Different steps in the generation of new vascular segments by intussusceptive angiogenesis (arrows) are illustrated in (c) and (d). Protrusion of a capillary pillar from opposite sides of the vessel wall into the vascular lumen is followed by perforation of the vessel, ultimately, splitting into two new vessels (arrows). Representative light microscopic images indicate the pulmonary vascular proliferation in $\mathrm{CBDL}$, resulting from these angiogenic processes (e, $\mathbf{f}$, arrows represent vessels).

flow. In the setting of PHT, this vein opens, becomes distended and redirects/shunts blood from the portal venous system into the lower esophageal veins and, ultimately, to areas with lower venous pressures (ie, superior vena cava). ${ }^{28}$ Therefore, the coronary vein was clearly visible on the $\mu$-CT images of the CBDL and PPVL animals, but absent in the SO rats (except in one SO rat, although with a negligible diameter, and therefore excluded during the CFD analysis). The highest WSS values were observed in the PPVL model (WSSav, 68.3 $\pm 24.5 \mathrm{~Pa}$; WSS95\%, 251.0 $\pm 94.2 \mathrm{~Pa}$ ) and the CBDL model (WSSav, 5.6 $\pm 1.1 \mathrm{~Pa}$; WSS95\%, $13.4 \pm 2.3 \mathrm{~Pa}$ ) and were significantly higher in comparison with $\mathrm{SO}$ rats (WSSav, $1.5 \pm 0.3 \mathrm{~Pa}$; WSS95\%, $3.8 \pm 0.6 \mathrm{~Pa}$ ) (respectively, $P<0.05$ and $P<0.05$ both $v s$ sham), indicating the same trend as previously derived from the dimensional analysis. Interestingly, $\mathrm{CFD}$ analysis was able to differentiate $\mathrm{CBDL}$ and PPVL rats according to their WSS $(P=0.02)$. Areas with higher WSS levels were located at the dorsal wall of flow splits and around small bulges in the portal vein's contours, in particular, around the ligation in the PPVL model. Variations in OSI, a marker of endothelial function disturbance, are plotted for the PPVL model in Figure 7 (data not shown for CBDL and sham).

Special care was taken when analyzing the PPVL model. PPVL was associated with numerous collaterals bypassing the ligation. As described above, CFD analysis was first performed without these collateral branches, but in a second calculation, these results were compared with the CFD, including the larger collaterals (Figure 6, calculations were performed in two PPVL rats). Importantly, the inclusion of collateral vessels in PPVL rats resulted in lower WSS values in comparison with the CFD analysis without collaterals (mean 

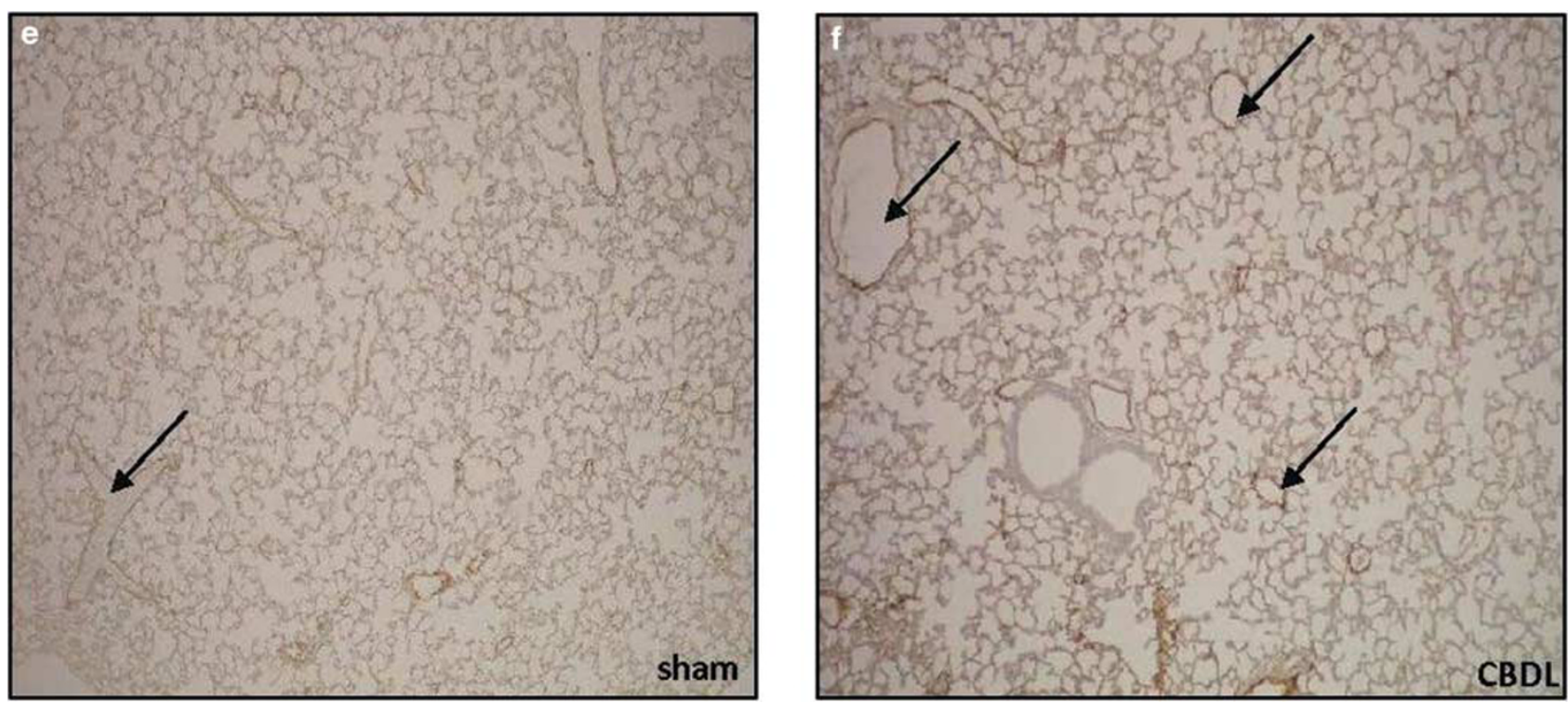

Figure 4 Continued.

\begin{tabular}{|l|c|c|c|}
\hline & $P P V L$ & $C B D L$ & Sham \\
\hline $\begin{array}{l}\text { Doppler Velocity } \\
\text { pattern }\end{array}$ & \\
& & & \\
\hline $\begin{array}{l}\text { Mean Flow } \\
\pm \text { sem }\end{array}$ & $1.54 \pm 0.15$ & $1.59 \pm 0.18$ & $0.91 \pm 0.05^{\mathrm{a}, \mathrm{b}}$ \\
\hline
\end{tabular}

Figure 5 Flow distribution pattern in different animal models of PHT and cirrhosis. Mean flow (ml/min) averaged over 20 cardiac cycles and averaged over three mice. (a) Sham vs PPVL, $P<0.05$, (b) Sham vs CBDL, $P<0.05$. The higher portal inflow in the PPVL and CBDL animals results from the splanchnic hyperemia in these models due to low splanchnic arteriolar resistance.

decrease $60.7 \%)$, but they still remained higher than those values in SO rats $(P=0.08)$. Owing to the slithering course and the high flow rates of the collaterals, the WSS in these shunts was increased.

As expected, the highest WSS was exerted during systole, but it remained different from zero during diastole. The WSS distribution in PPVL at different time steps of the cardiac cycle is displayed in Figure 8.

\section{DISCUSSION}

Vascular corrosion casting is an established and well-described method of anatomical preparation that has been used since the middle ages for imaging of hollow organs and vascular systems. Although this technique was initially used for macroscopic purposes, corrosion anatomy is contemporarily applied in both stereo and scanning electron microscopic procedures. ${ }^{6}$
Table 1 Dimensional analysis in the portal vein of different animal models

\begin{tabular}{lccc}
\hline & Sham & CBDL & PPVL \\
\hline Inlet diameter portal vein $(\mathrm{mm})$ & $2.8 \pm 0.07$ & $2.5 \pm 0.17$ & $2.6 \pm 0.004$ \\
Reynolds number $(\mathrm{Re})^{\mathrm{a}}$ & $115 \pm 17$ & $285 \pm 27^{* *}$ & $237 \pm 6^{*}$ \\
${\text { Womersley number }(\alpha)^{\mathrm{b}}}^{\text {WSS }(\mathrm{Pa})^{\mathrm{c}}}$ & $2.9 \pm 0.2$ & $2.6 \pm 0.3$ & $4.1 \pm 0.3$ \\
& $1.4 \pm 0.2$ & $5.3 \pm 1.4^{* * *}$ & $3.2 \pm 0.09^{* * *}$
\end{tabular}

\footnotetext{
${ }^{\mathrm{a}}$ The Re allows the assessment of whether the flow is laminar $(\operatorname{Re}<2000)$, turbulent $(\operatorname{Re}>2500)$ or in a transition phase $(2000 \leq \operatorname{Re} \geq 2500)$.

${ }^{\mathrm{b}}$ The Womersley number $(\alpha)$ takes the pulsatile blood flow into account. When $\alpha$ is low, the flow can be estimated by Poiseuille's law.

'The WSS is computed using Poiseuille's law.

${ }^{*} P<0.001$ vs sham.

${ }^{*} P<0.01$ vs sham.

$* * * P<0.05$ vs sham.
} 

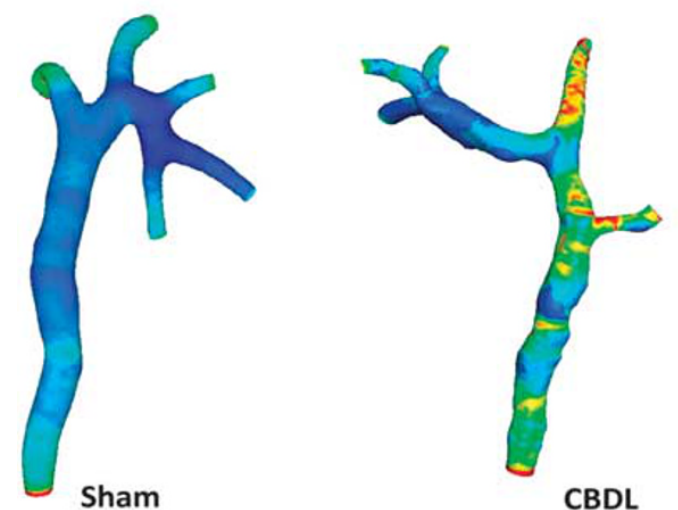

CBDL

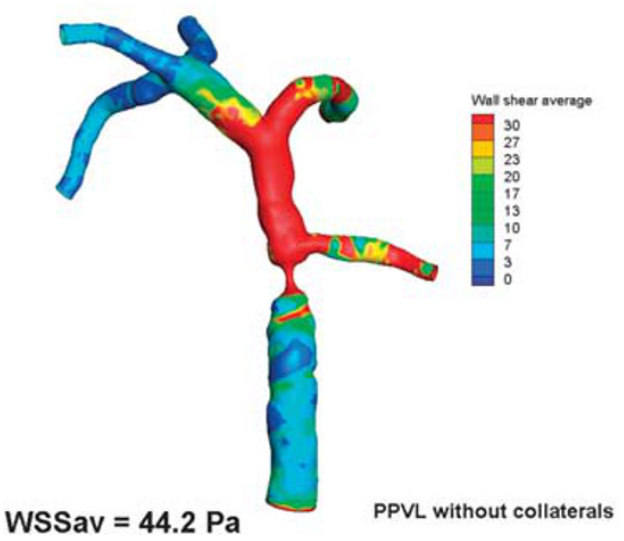

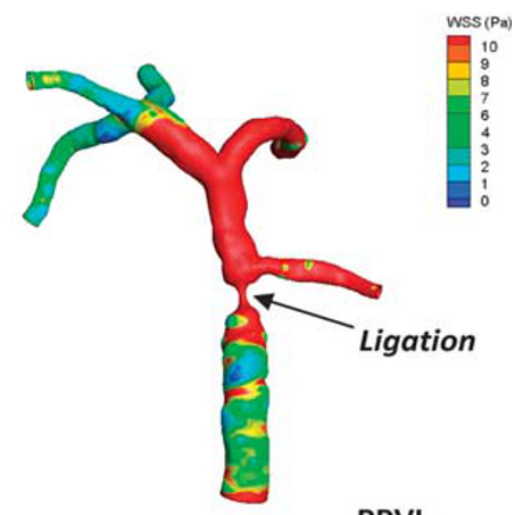

PPVL

Figure 6 Wall shear stress (WSS) in the portal vein in the different animal models. Upper panel: Spatial distribution of the WSS in the portal vein, time-averaged over a cardiac cycle. The highest WSS values are observed in the PPVL model in comparison with CBDL and sham. Areas with high WSS were located at the dorsal wall of flow splits and around small bulges in the portal vein's contours, clearly demonstrated around the ligation in the PPVL model (arrow). Lower panel: WSS distribution in the portal vein of PPVL with or without collaterals. The formation of portosystemic collaterals decreased the WSS in the portal vein in comparison with the situation without collaterals. However, normalization of the portal venous WSS was not reached (calculation was executed in two PPVL rats). Owing to the slithering course and the high flow rates of the collaterals, the WSS in these shunts was increased in an effort to relieve the portal vein.

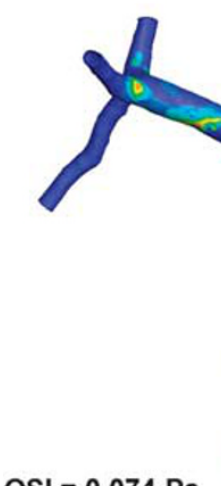

OSI $=0.074 \mathrm{~Pa}$
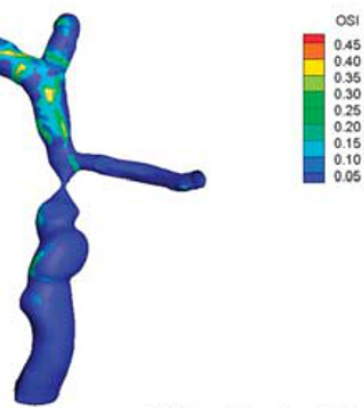

PPVL without collaterals

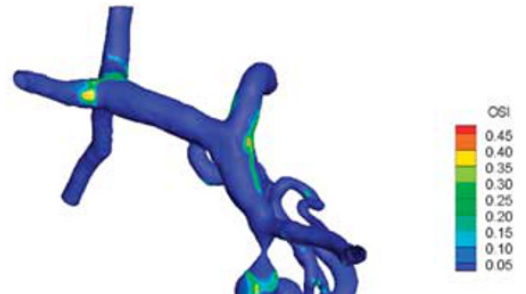

$O S I=0.026 \mathrm{~Pa}$

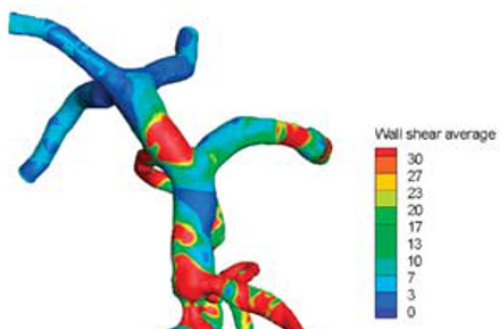

PPVL with collatera

Figure 7 Oscillatory shear index (OSI) distribution, as a marker of endothelial function disturbance, in the portal vein of PPVL with or without collaterals. Consistent with the results of WSS distribution in PPVL, the formation of portosystemic shunts in PPVL reduced the average OSI by 55\% in comparison with the noncollateral configuration (calculation with and without collaterals was executed in two PPVL rats).

The first part of this study provides detailed morphological information on the microvasculature in mice models of PHT and cirrhosis. To our knowledge, this is the first study that describes the vascular changes in CBDL cirrhosis. Many features, recently described using molecular techniques in basic hepatology research are hereby confirmed using these 

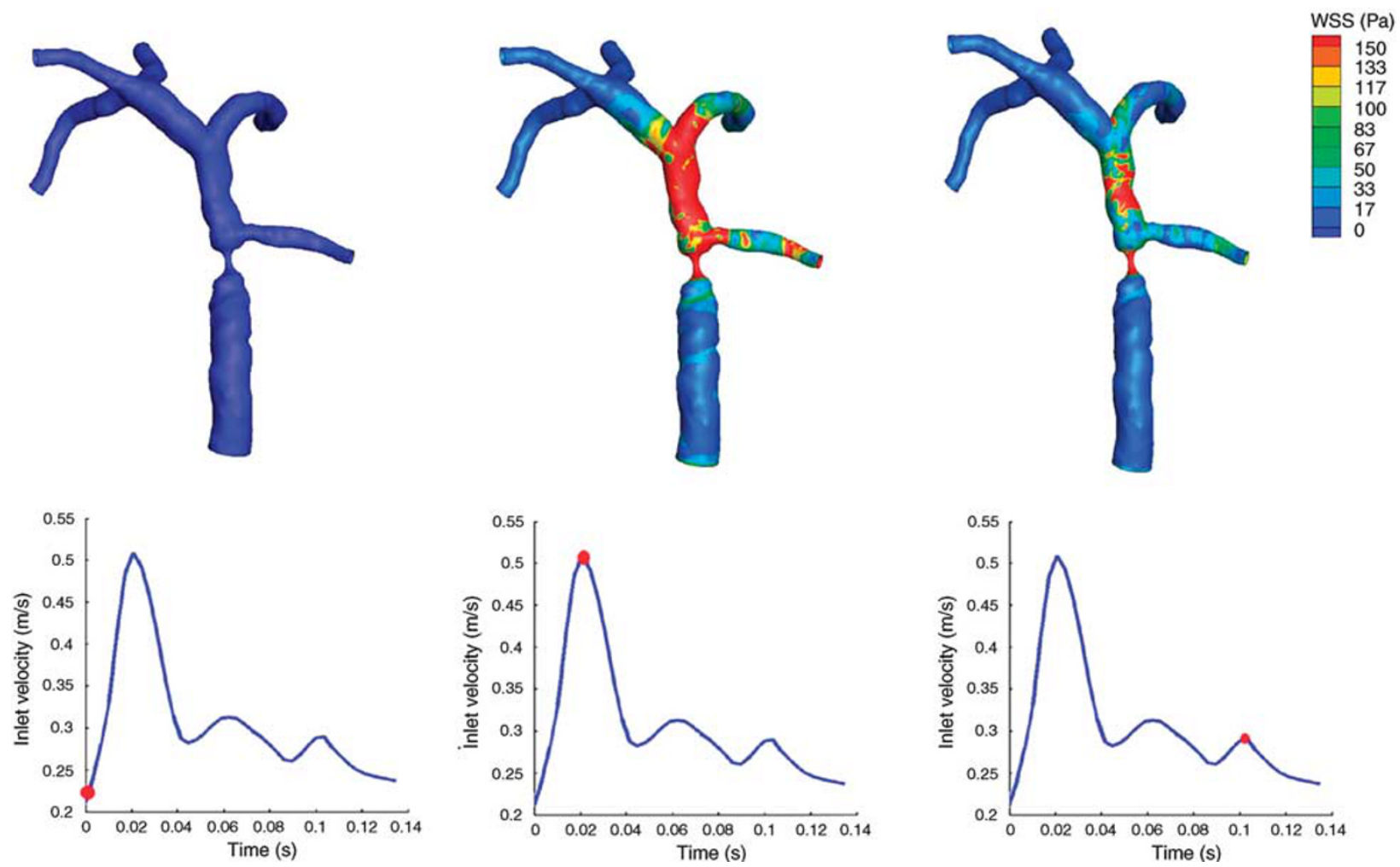

Figure 8 WSS distribution at different time steps of the cardiac cycle in the PPVL model. The highest WSS was exerted during systole, but remained different from zero during diastole.

vascular casts, such as neoangiogenesis and intussusceptive angiogenesis in different vascular beds of portal hypertensive and cirrhotic mice. In addition, the technique of vascular corrosion casting is an adequate alternative for visualizing angiogenesis and vessel morphology to classical methods such as immunohistochemistry. Both casts and immunohistochemistry can act as complementary techniques as demonstrated in this study in which the morphological changes described on the casts are further substantiated by immunohistochemistry of blood vessels. As recently reported in other studies, ${ }^{29-31}$ the casting methodology is sensitive enough to observe sequential microvascular changes after pharmacological or surgical intervention. Morphometrical quantitative studies can be performed on vascular corrosion casts, but are frequently hampered by their inherent dependency on the casting medium properties (eg, shrinkage of Batson's polymer ${ }^{32}$ ). Therefore, these quantification have to be interpreted with caution.

Several studies have indicated increased angiogenesis as a key structural feature in the splanchnic, hepatic and pulmonary territory of portal hypertensive and cirrhotic rats. ${ }^{29,33,34}$ Consistent with these reports, the present results are also indicative of splanchnic vascular proliferation in PPVL/CBDL mice (as seen with stereomicroscopic examination and scanning electron microscopy). Electron microscopic evaluation revealed the different steps in the generation of new vascular segments by intussusceptive angiogenesis (splitting angiogenesis) in splanchnic organs of portal hypertensive mice and in the lungs of cirrhotic mice. ${ }^{25}$ First, a capillary pillar protrudes from the opposite sides of the vessel wall into the vascular lumen, followed by perforation of the vessel. These findings clearly occurred more frequently in pathological animals in comparison with normal healthy mice. In addition, sprouting angiogenesis, defined as tubular extensions from a preexisting vessel forming entirely new vessels, was also identified in diseased animals and was barely present in healthy animals.

Electron microscopy of cirrhotic livers showed marked vascular abnormalities. We hypothesize that changes in blood flow due to significant rheological factors (eg, thrombosis, vasoconstriction or fibrosis) cause narrowing of the sinusoidal lumen, resulting in the obliteration of the normal trabecular pattern of Christmas tree appearance and the shunting of blood in the portocentral collaterals. Furthermore, these collaterals exhibited a leaky appearance, reflecting changes in vascular permeability and yielding easily to leakage for the casting media.

Vascular casting has been used in a variety of pathologies, including human cirrhotic livers ${ }^{35}$ and $\mathrm{CCL}_{4}$-induced experimental cirrhosis. ${ }^{36,37}$ In contrast to $\mathrm{CCL}_{4}$ cirrhosis, CBDL was associated with a more pronounced disruption of the liver architecture on vascular casts. Previous studies have confirmed a markedly lower portal sinusoidal flow in CBDL rats in comparison with those with $\mathrm{CCL}_{4}$ 
cirrhosis leading to more sinusoidal narrowing and subsequent obliteration of casts in this study. ${ }^{38}$ In this model of biliary obstruction, different factors (in particular, macronodular cirrhosis, ductular proliferation, biloma formation and prominent inflammatory infiltrates ${ }^{13}$ ) contributed to more extensive microvascular and hemodynamic changes in comparison with $\mathrm{CCL}_{4}$ micronodular cirrhosis, leading to an amorphous and deformed vessel organization on vascular casts.

The main novelty reported in this study is the capacity to measure the WSS in the portal vein of different rat models of PHT and cirrhosis. A large body of evidence has demonstrated the influence of WSS on vascular endothelial cells and vascular smooth muscle cells in initiating different events, such as flow-mediated vasodilatation, vascular remodeling and cellular movement. ${ }^{9}$ To our knowledge, no techniques have been developed to quantify WSS in the portal vein in laboratory animals. Our current knowledge with regard to the mechanisms by which shear stress affects the portal vein is derived from liver perfusion models and in vitro experiments in which monolayers of endothelium are exposed to a well-defined, simple fluid dynamic environment. ${ }^{39-42}$ In contrast, CFD methods based on 3D vessel reconstructions in the arterial vascular bed are widely used in the cardiovascular research field and recently provided prognostic data. Our findings demonstrate that deriving WSS using CFD methods is a feasible technique in PHT and cirrhosis, enabling the differentiation between animal models. First, a dimensional analysis was performed, yielding larger WSS values in CBDL/PPVL vs sham animals, as shown in Table 1. The higher portal inflow in the PPVL and CBDL animals resulted from the splanchnic hyperemia in these models, associated with the low splanchnic arteriolar resistance. ${ }^{43}$ The Womersley parameters were rather small, indicating an almost quasistatic behavior and supporting the use of Poiseuille's law to detect a trend in WSS. The Reynolds numbers were doubled in PPVL and CBDL in comparison with sham animals, as a consequence of the higher inlet portal venous flow, even though the inlet portal vein diameters were rather similar in all groups (Table 1).

Additionally, a CFD calculation was performed to study the spatial and temporal WSS distributions in more detail. Both analyses showed a significant difference in temporally and spatially averaged WSS (WSSav), as well as the 95th percentile of temporally averaged WSS (WSS95\%) between sham/CBDL/PPVL animals, with, not unexpectedly, the highest values in the PPVL model. The areas adjacent to the ligation and the dorsal walls of flow splits were the most exposed locations. The highest WSS values in the PPVL model were the consequence of both concomitant changes in flow and manifest variabilities in geometry, whereas the discrimination between sham and CBDL was mainly attributable to flow differences. In agreement with this explanation, CFD analysis was able to differentiate CBDL and PPVL rats according to their WSS, whereas this was not possible in the dimensional analysis, which takes only flow differences and inlet diameters into account.

In addition, the formation of portosystemic collaterals was linked to the WSS distribution in this study. Portosystemic collateral vessels are formed in an attempt to decompress the portal vein by redirecting a fraction of the portal venous flow through these vessels. This results in lower WSS values in the portal vein and higher values in the collaterals (both by higher flow through these vessels and their twisted course in geometry). Although we observed that these shunts are beneficial in relieving the portal vein, as the WSSav was reduced by $60.7 \%(n=2)$, no normalization in WSS was attained.

Moreover, this study also addressed the distribution of OSI values in the portal vein in the PPVL model. As endothelial cells are susceptible to the magnitude and changes in WSS direction, areas with high WSSav and OSI values are indirect markers for endothelial disturbances in these PPVL rats. ${ }^{12,23}$

Nevertheless, some limitations in the current work might have influenced the obtained numerical results. First, the ideal casting medium does not exist. The physical-technical limitations of the casting medium (particularly viscosity and shrinkage) have to be taken into consideration when interpreting results, especially in morphometric and quantitative studies. Batson's polymer is known to shrink slightly, resulting in a small underestimation of the vessel diameters on casting in comparison with in vivo imaging. ${ }^{6}$ However, this phenomenon will not influence the general conclusion of this study. Second, the flow splits in both main portal vein branches used in CFD analysis were estimated using Murray's law. Using an ultrasonic blood flow probe, it is technically very difficult to perform flow measurements around the intrahepatic course of the portal branches in rats. Estimating WSS experimentally from velocity profiles recorded noninvasively by means of MRI or ultrasound is, to some extent, easier in a human setting. Although keeping in mind the limitations of translating results from animal models to practice, studying the WSS in different clinical settings in humans may be of interest in characterizing the fluid dynamic environment of the vessel wall in vivo. Third, the performed numerical model cannot include all physiological parameters for pulsatile flow and non-Newtonian behavior of the blood. ${ }^{44}$ All these aspects were thoroughly investigated in arterial vascular beds in recent studies. ${ }^{45,46}$ Nevertheless, in comparison with arterial WSS studies, pulsatile flow can be expected to be of minor importance in a lower-pressure vessel similar to the portal vein. Finally, the use of CFD methods permits only a simulation of the real geometric and hemodynamic differences between models, as it is limited by excessive complexity of the geometry. Besides computational constraints, no flow measurements were possible across all accessory collateral branches in the PPVL model using the current methodology in this paper. Likewise, we were forced to remove the smaller collateral branches in the PPVL model, while preserving the larger ones. However, in a human setting, accurate replication of the in vivo 
hemodynamics across the (larger) collaterals (by means of MRI) must be taken into account.

In conclusion, this study showed for the first time that vascular casting has an important role, not only in the morphological evaluation of different animal models of PHT and cirrhosis, but also in defining the biological response of the portal vein wall to hemodynamic changes. CFD in 3D geometries can be used to describe the spatial and temporal variations in WSS and to better understand the forces affecting mechanotransduction in the vascular endothelium and smooth muscle cells. In analogy to cardiovascular fluid mechanics, the synergy between CFD modeling and noninvasive imaging of the portal vein can be expected to grow in the future, thereby permitting possible extensions in the clinical domain.

Supplementary Information accompanies the paper on the Laboratory Investigation website (http://www.laboratoryinvestigation.org)

\section{ACKNOWLEDGEMENTS}

We thank Femke Heindryckx for her input in the illustrations of this paper. This work was supported by a grant from the Fund for Scientific Research (Aspirant mandaat-FWO Vlaanderen, 1.1.466.09.N.0, to Christophe Van Steenkiste).

\section{DISCLOSURE/CONFLICT OF INTEREST}

The authors declare no conflict of interest.

1. Roberts SE, Goldacre MJ, Yeates D. Trends in mortality after hospital admission for liver cirrhosis in an English population from 1968 to 1999. Gut 2005;54:1615-1621.

2. Shah V. Therapy for portal hypertension: what is our pipeline? Hepatology 2009;49:4-5.

3. Fernandez $M$, Semela $D$, Bruix J, et al. Angiogenesis in liver disease. J Hepatol 2009;50:604-620.

4. Ciura VA, Lee MJ, Schemmer DC. MRA: current applications in body vascular imaging. Can Assoc Radiol J 2009;60:133-142.

5. Mondy WL, Cameron D, Timmermans JP, et al. Micro-CT of corrosion casts for use in the computer aided design of microvasculature. Tissue Eng Part C Methods 2009;14:729-738.

6. Konerding MA. Scanning electron microscopy of corrosion casting in medicine. Scanning Microsc 1991;5:851-865.

7. Wei W, Popov V, Walocha JA, et al. Evidence of angiogenesis and microvascular regression in autosomal-dominant polycystic kidney disease kidneys: a corrosion cast study. Kidney Int 2006;70:1261-1268

8. Papaioannou TG, Karatzis EN, Vavuranakis M, et al. Assessment of vascular wall shear stress and implications for atherosclerotic disease. Int J Cardiol 2006;113:12-18.

9. Malek AM, Izumo S, Alper SL. Modulation by pathophysiological stimuli of the shear stress-induced up-regulation of endothelial nitric oxide synthase expression in endothelial cells. Neurosurgery 1999;45:334-344.

10. Irace C, Cortese C, Fiaschi E, et al. Wall shear stress is associated with intima-media thickness and carotid atherosclerosis in subjects at low coronary heart disease risk. Stroke 2004;35:464-468.

11. Suo J, Ferrara DE, Sorescu D, et al. Hemodynamic shear stresses in mouse aortas: implications for atherogenesis. Arterioscler Thromb Vasc Biol 2007;27:346-351.

12. Trachet B, Swillens A, Van Loo D, et al. The influence of aortic dimensions on calculated wall shear stress in the mouse aortic arch. Comput Methods Biomech Biomed Engin 2009;12:491-499.

13. Geerts AM, Vanheule $E$, Praet $M$, et al. Comparison of three research models of portal hypertension in mice: macroscopic, histological and portal pressure evaluation. Int J Exp Pathol 2008;89:251-263.

14. Fernandez M, Vizzutti F, Garcia-Pagan JC, et al. Anti-VEGF receptor-2 monoclonal antibody prevents portal-systemic collateral vessel formation in portal hypertensive mice. Gastroenterology 2004;126:886-894.
15. Mejias M, Garcia-Pras E, Tiani C, et al. Beneficial effects of sorafenib on splanchnic, intrahepatic, and portocollateral circulations in portal hypertensive and cirrhotic rats. Hepatology 2009;49:1245-1256.

16. Callewaert BL, Loeys BL, Casteleyn C, et al. Absence of arterial phenotype in mice with homozygous slc2A10 missense substitutions. Genesis 2008;46:385-389.

17. Feintuch $A$, Ruengsakulrach $P$, Lin $A$, et al. Hemodynamics in the mouse aortic arch as assessed by MRI, ultrasound, and numerical modeling. Am J Physiol Heart Circ Physiol 2007;292:H884-H892.

18. Lee $D$, Chen JY. Numerical simulation of steady flow fields in a model of abdominal aorta with its peripheral branches. J Biomech 2002;35:1115-1122.

19. Teleuhan N. Delineation of right and left lobe of the liver accessed by 3-dimensional CT images. Medical Journal of Hiroshima University 2003;51:33-40.

20. Greve JM, Les AS, Tang BT, et al. Allometric scaling of wall shear stress from mice to humans: quantification using cine phase-contrast MRI and computational fluid dynamics. Am J Physiol Heart Circ Physiol 2006;291:H1700-H1708.

21. Papaharilaou Y, Doorly DJ, Sherwin SJ. The influence of out-of-plane geometry on pulsatile flow within a distal end-to-side anastomosis. J Biomech 2002;35:1225-1239.

22. Ku DN, Giddens DP, Zarins CK, et al. Pulsatile flow and atherosclerosis in the human carotid bifurcation. Positive correlation between plaque location and low oscillating shear stress. Arterioscler Thromb Vasc Biol 1985;5:293-302.

23. Huo Y, Guo X, Kassab GS. The flow field along the entire length of mouse aorta and primary branches. Ann Biomed Eng 2008;36:685-699.

24. Burri PH, Tarek MR. A novel mechanism of capillary growth in the rat pulmonary microcirculation. Anat Rec 1990;228:35-45.

25. Clauss $M$, Breier G. Mechanisms of Angiogenesis. Birkhäuser Verlag: Basel-Boston-Berlin, 2005.

26. Fallon MB, Abrams GA, McGrath JW, et al. Common bile duct ligation in the rat: a model of intrapulmonary vasodilatation and hepatopulmonary syndrome. Am J Physiol 1997;272(4 Pt 1):G779-G784.

27. Schraufnagel $D E$, Malik $R$, Goel $V$, et al. Lung capillary changes in hepatic cirrhosis in rats. Am J Physiol 1997;272(1 Pt 1):L139-L147.

28. Arguedas MR. The critically ill liver patient: the variceal bleeder. Semin Gastrointest Dis 2003;14:34-38.

29. Van Steenkiste C, Geerts A, Vanheule E, et al. Role of placental growth factor in mesenteric neo-angiogenesis in a mouse model of portal hypertension. Gastroenterology 2009;137:2112-2124.

30. Van Steenkiste C, Ribera J, Geerts A, et al. Inhibition of placental growth factor activity reduces the severity of inflammation, fibrosis and portal hypertension in cirrhotic mice. unpublished data.

31. Heindryckx F, Mertens K, Charette N, et al. Kinetics of angiogenic changes in a new mouse model for hepatocellular carcinoma. unpublished data.

32. Kratky RG, Roach MR. Shrinkage of Batson's and its relevance to vascular casting. Atherosclerosis 1984;51:339-341.

33. Tugues $\mathrm{S}$, Fernandez-Varo G, Munoz-Luque J, et al. Antiangiogenic treatment with sunitinib ameliorates inflammatory infiltrate, fibrosis, and portal pressure in cirrhotic rats. Hepatology 2007;46: 1919-1926.

34. Zhang J, Luo B, Tang L, et al. Pulmonary angiogenesis in a rat model of hepatopulmonary syndrome. Gastroenterology 2009;136: 1070-1080.

35. Baldus WP, Hoffbauer FW. Vascular changes in the cirrhotic liver as studied by injection technic. Am J Dig Dis 1963;8:689-700.

36. Gaudio E, Pannarale L, Ripani $M$, et al. The hepatic microcirculation in experimental cirrhosis. A scanning electron microscopy study of microcorrosion casts. Scanning Microsc 1991;5:495-502.

37. Gaudio E, Pannarale L, Onori P, et al. A scanning electron microscopic study of liver microcirculation disarrangement in experimental rat cirrhosis. Hepatology 1993;17:477-485.

38. Van de Casteele M, Sagesser H, Zimmermann $\mathrm{H}$, et al. Characterisation of portal hypertension models by microspheres in anaesthetised rats: a comparison of liver flow. Pharmacol Ther 2001;90:35-43.

39. Pastor $\mathrm{CM}$, Hadengue A. Shear stress modulates the vascular tone in perfused livers isolated from normal rats. Hepatology 2000;32 (4 Pt 1):786-791.

40. Macedo MP, Lautt WW. Shear-induced modulation of vasoconstriction in the hepatic artery and portal vein by nitric oxide. Am J Physiol 1998;274(2 Pt 1):G253-G260. 
41. Mittal MK, Gupta TK, Lee FY, et al. Nitric oxide modulates hepatic vascular tone in normal rat liver. Am J Physiol 1994;267(3 Pt 1):G416G422.

42. Shah V, Haddad FG, Garcia-Cardena G, et al. Liver sinusoidal endothelial cells are responsible for nitric oxide modulation of resistance in the hepatic sinusoids. J Clin Invest 1997;100: 2923-2930.

43. Colle I, Geerts AM, Van Steenkiste C, et al. Hemodynamic changes in splanchnic blood vessels in portal hypertension. Anat Rec (Hoboken) 2008;291:699-713.

\section{APPENDIX A}

\section{Hemodynamic Parameters}

Dimensional Analysis

The Reynolds $(\mathrm{Re})$ and Womersley $(\alpha)$ numbers are defined, respectively, as follows: ${ }^{22}$

$$
\operatorname{Re}=V D / v
$$

where $V$ is the mean inlet velocity; $D$, the inlet diameter; and $v$, the kinematic blood viscosity.

$$
\alpha=R \sqrt{ }(\omega / v)
$$

where $R$ is the inlet radius; $\omega$, the angular frequency of heart beats; and $v$, the kinematic blood viscosity.

When $\alpha$ is small $(<1)$, the frequency of pulsations is sufficiently low to develop a parabolic velocity profile during each cycle, and the flow will be nearly in phase with the pressure gradient, that is, laminar flow. WSS is subsequently calculated using Poiseuille's law. When $\alpha$ is large $(>10)$, the frequency of pulsations is sufficiently large, the velocity profile is relatively flat and the mean flow lags the pressure gradient by about $90^{\circ}$.

WSS $\left(\tau_{\text {Pois }}\right)$ is first estimated using Poiseuille's law. However, this is only valid in laminar steady flow conditions in a straight cylindrical tube:

$$
\tau_{\text {Pois }}=8 \mu V / D
$$

44. Goubergrits L, Wellnhofer E, Kertzscher U, et al. Coronary artery WSS profiling using a geometry reconstruction based on biplane angiography. Ann Biomed Eng 2009;37:682-691.

45. LaDisa JF, Olson LE, Guler I, et al. Circumferential vascular deformation after stent implantation alters wall shear stress evaluated with timedependent 3D computational fluid dynamics models. J Appl Physiol 2005;98:947-957.

46. Myers JG, Moore JA, Ojha M, et al. Factors influencing blood flow patterns in the human right coronary artery. Ann Biomed Eng 2001;29:109-120.

where $\mu$ is the dynamic blood viscosity; $v \times \rho$, with $\rho$ the density of blood; $V$, the mean inlet velocity; and $D$, the inlet diameter.

\section{CFD Analysis}

In each CFD model, the time-averaged WSS distribution (WSSav) was computed as

$$
\text { WSSav }=\frac{1}{T} \int_{0}^{T} \tilde{\tau} \mathrm{d} t
$$

where $T$ is the cardiac cycle time period $(0,1 \mathrm{~s})$; and $\widetilde{\tau}$, the WSS vector

To obtain a measure of the oscillatory motion of the WSS vector, the OSI has been calculated. As both shear vector magnitude and direction change with time in a continuous manner, the OSI is defined as follows:

$$
\text { OSI }=\frac{1}{2}\left[1-\frac{\left|\frac{1}{T} \int_{0}^{T} \widetilde{\tau} \mathrm{dt}\right|}{\frac{1}{T} \int_{0}^{T}|\tilde{\tau}| \mathrm{d} t}\right]
$$

The range of values for the OSI is $0<\mathrm{OSI}<0.5$, where 0 corresponds to unidirectional shear flow and 0.5 corresponds to purely oscillatory motion. 